\title{
Detection of Free Cancer Cells in Pelvic Lavage with Double Immunocytochemistry at Rectal Cancer Surgery
}

\author{
CSABA KINDLER ${ }^{1}$, KENNETH SMEDH $^{2,3}$, ABBAS CHABOK ${ }^{2,3}$, \\ JAYANT SHETYE ${ }^{1}$, GEORGE DAFNIS $^{4}$ and MAZIAR NIKBERG ${ }^{2,3}$ \\ Departments of ${ }^{1}$ Pathology, ${ }^{2}$ Surgery and ${ }^{3}$ Centre for Clinical Research of Uppsala University, \\ Vastmanland's Hospital Vasteras, Vasteras, Sweden; \\ ${ }^{4}$ Department of Surgery, Mälar Hospital, Eskilstuna, Sweden
}

\begin{abstract}
Background/Aim: The aim of the present study was to describe a double immunocytochemical staining method for detecting free cancer cells after rectal cancer surgery and to evaluate their extent and prognostic role. Materials and Methods: Immunocytochemistry was performed using antibodies against cytokeratin 20/caudaltypehomeobox transcription factor 2 (CDX2) and mucin glycoprotein-2 (MUC2)/p53 protein. The study included 29 patients with infraperitoneal rectal cancer who underwent bowel resection and four controls. The pelvic lavage was retrieved at the start of laparotomy, after total mesorectal excision and after abdominal lavage with sterile water. Results: Free cancer cells were detected with the double immunocytochemical method in the two controls with carcinomatosis and one control with sigmoidal cancer. None of the patients with rectal tumours had presence of free cancer cells. Conclusion: Immunocytochemical analysis of peritoneal lavage was feasible and negative in patients with infraperitoneal rectal cancer. Further studies are encouraged to investigate the clinical relevance in cases with free cancer cells after incomplete total mesorectal excision.
\end{abstract}

Advances in oncological treatment and meticulous specimenoriented total mesorectal excision (TME) have improved local and distant tumour control in patients with rectal cancer. An incomplete mesorectal excision has been reported to occur in up to $57 \%$ of patients undergoing rectal cancer resection and occurs more commonly after abdomino-

This article is freely accessible online.

Correspondence to: Maziar Nikberg, MD, Ph.D., Department of Surgery, Västmanland's Hospital Västerås 72189 Västerås, Sweden. Tel: +46 21173000, Fax: +46 21175347, e-mail: maziar.nikberg@ltv.se

Key Words: Rectal cancer, peritoneal cancer cells, immunocytochemistry, immunocytology recurrence. perineal excision (APE) than after anterior resection (1). Involvement of the circumferential resection margin (CRM) has, in numerous studies, been correlated to an increased risk of local recurrence $(2,3)$. There are, however, studies demonstrating no correlation of CRM involvement and local recurrence, implying that involvement of the CRM results in local recurrences only when the mesorectal excision is incomplete $(4,5)$. Recurrence after incomplete mesorectal resection with defects in the mesorectal fascia (MRF) could be a result of direct tumour spillage or disruption of tumourcontaining blood or lymphatic vessels in the MRF.

In order to detect cancer cells in peritoneal lavage, different methods have been used, resulting in a wide variety of reported positive rates. In most studies on colorectal cancer, $5-25 \%$ of patients had peritoneal lavage positive for cancer cells (6-8). The most commonly used method is with conventional Giemsa staining and might be sufficient in the presence of peritoneal carcinomatosis but is insensitive in the detection of free cancer cells in peritoneal lavage in the absence of peritoneal metastases (9). The polymerase chain reaction technique which detects Kirsten ras 2 (K-RAS) mutations can be used to detect tumour cells in peritoneal lavage; however, only $15-33 \%$ of rectal cancers have a mutation in the $K-R A S$ gene (10). Immunocytochemical analysis of several different proteins (cytokeratin 20, carbohydrate antigen19-9, epithelial cell adhesion molecule BER-EP4, C1P83 antigen, LU5 antigen) has also been used, however, with inconsistent tumour cell detection rates (11).

In order to increase the sensitivity of the cytological detection of cancer cells, a double immunocytochemical staining method can be used. Tumour cells are then identified with two different markers applied simultaneously to the same sample. To our knowledge, use of double immunocytochemistry of pelvic lavage has not been reported.

A positive peritoneal lavage in colorectal cancer is a negative prognostic factor (11). However, the role of pelvic lavage as a surgical quality marker in infraperitoneal rectal cancer is unclear. We hypothesised that pelvic lavage would 
be negative in all patients with infraperitoneal rectal cancer and, furthermore, that pelvic lavage would be negative after complete TME without injury to the mesorectal fascia.

The aim of the study was to describe the double immunocytochemical staining method and to evaluate the occurrence and prognostic role of free cancer cells in pelvic lavage in patients with infraperitoneal rectal cancer.

\section{Materials and Methods}

Patients and surgery. Between 2012 and 2014, 29 consecutive patients with rectal cancer planned for anterior resection, APE or Hartmann's procedure were included prospectively at the Västmanland's Hospital Västerås, Sweden. In addition, two patients with peritoneal carcinomatosis of colorectal cancer origin, two with sigmoidal cancer with macroscopically suspected serosal involvement and one with a distal rectal cancer that was not resected were included as controls.

A midline laparotomy was performed in all cases and the surgeon's assessment of serosal involvement of the tumour was registered. Prior to the start of the dissection, the pelvis was washed with $250 \mathrm{ml}$ of saline solution which was thereafter completely aspirated as sample 1. After completion of the TME, performed by three experienced colorectal surgeons, the surgical field was once again washed with $250 \mathrm{ml}$ of saline solution that was aspirated completely as sample 2 . In all cases the APE was performed as an extralevator APE.

The pelvic and abdominal cavity were rinsed with two litres of heated $\left(37^{\circ} \mathrm{C}\right)$ sterile water for 5 minutes in all cases after tumour resection. Following the removal of all sterile water, once again $250 \mathrm{ml}$ of saline solution were instilled in the pelvic cavity and aspirated as sampl -3 .

The completeness of the MRF was graded using the Quirke classification into mesorectal, intramesorectal and muscularis propria planes (1).

All patients were routinely monitored with computed tomography of the thorax and abdomen year one and three after surgery and by clinical examination, including rigid sigmoidoscopy, yearly during the first three years. A local recurrence was defined as the presence of tumour clinically, radiologically or histologically in the pelvis or perineum irrespective of the presence of distant metastases.

The date and cause of death were acquired from the Swedish Cause of Death Registry.

Sample treatment. The aspirated fluid was centrifuged at 1,200 $\times g$ for $10 \mathrm{~min}$ and the supernatant was poured off. The pellet was resuspended in 3-8 $\mathrm{ml}$ of a hemolytic agent (ThinPrep CytoLyt Solution; Hologic Ltd., Manchester, UK). After $10 \mathrm{~min}$, the sample was centrifuged again at $1200 \times g$ for $10 \mathrm{~min}$. Depending on the amount of blood, the process was repeated. The pellet was finally re-suspended in EnVision FLEX Wash Buffer (20x) (Dako, Glostrup, Denmark) to a volume of $600 \mu$. The sample was placed in six chambers $(100 \mu \mathrm{l}$ in each) and was centrifuged at $30 \times g$ for $5 \mathrm{~min}$. All the slides were air-dried and kept at $-70^{\circ} \mathrm{C}$ wrapped in paraffin film. Six slides were created in order to create an optimal cell volume, but only three randomly selected slides were further analysed.

Immunocytochemistry was performed by using the Autostainer link FLEX system (Dako) with the help of PT link (Dako) for antigen retrieval. In order to identify tumour cells, a panel of four antibodies was used. Antibodies to the epithelial marker cytokeratin 20 (CK20. clone Ks20.8; Dako), which stains normal colorectal epithelia (12), together with the caudal-type Homeobox transcription factor 2 (FLEX Monoclonal Mouse Anti-Human CDX2 Clone DAK-CDX2; Dako), which stains the nuclei of normal colonic epithelium, were used in the first panel to detect cells of colorectal origin (13). Antibodies to mucin glycoprotein-2 (MUC2, clone Ccp58; Novocastra, Newcastle Upon Tyne, UK) and p53 protein (p53, clone DO-7; Dako) were used in the second panel to identify neoplastic change in cells of colorectal origin $(14,15)$. p53 and CDX2 stained the nuclei using 3,3'-diaminobenzidine (DAB) as chromogen while permanent red chromogen labelled the cytoplasmic markers CK20 and MUC2.

Slide 1 was stained with haematoxylin \& eosin. Slide 2 was stained a combination of antibodies to CK20 and CDX2. In cases with cytokeratin with/without CDX2-positive cells, slide 3 was stained with antibodies to MUC2 and p53.

Definitions. In sample 1, the presence of CK20/CDX2-positive cells was interpreted as a tumour cell as long as the bowel wall was intact/not transected. In samples 2 and 3, the addition of MUC2/p53 positivity with morphologically atypical cells was required to identify tumour cells. An isolated cytoplasmic fragment with weak CK20 or MUC2 staining or free nucleus with weak CDX2 or p53 staining was considered as an artefact.

\section{Results}

The clinical, surgical and histopathological characteristics of all patients are summarized in Table I.

All patients were staged pre-operatively with magnetic resonance imaging of the pelvis and computed tomography of the thorax/abdomen. In total, $15(52 \%)$ patients received preoperative radiotherapy and 7 (24\%) preoperative chemoradiotherapy. When concomitant chemotherapy was not administered, most patients underwent short-course radiotherapy ( 5 Gy $\times 5$ in 1 week) followed by immediate or delayed surgery (8 weeks after the completion of radiotherapy). All tumours were situated below the peritoneal reflection.

Controls. Sample 1 revealed cancer cells (atypical and CK20/CDX2-positive cells) in one of the two patients with sigmoidal cancer with macroscopically suspected serosal involvement (Figure 1A). There were no cancer cells in the control patient with infraperitoneal rectal tumour.

In the two patients with peritoneal carcinomatosis of colorectal cancer origin, the presence of cancer cells was confirmed by both H\&E staining (Figure 1B) and immunocytochemical analyses: CK20/CDX2 (Figure 1C) and MUC2/p53 (Figure 1D).

Patients who underwent resection for rectal cancer. Sample 1 contained a variable number of mesothelial cells. In one of the patients with rectal tumours, few cells stained positively for CK20/CDX2 (Figure 2A) and MUC2 (Figure 2B); 
Table I. Clinical, surgical and histopathological characteristics of patients with rectal cancer included in this study $(n=29)$.

\begin{tabular}{|c|c|c|}
\hline Characteristic & Subgroup & Value \\
\hline Age (range), years & & $71(46-81)$ \\
\hline \multirow[t]{2}{*}{ Gender, $\mathrm{n}(\%)$} & Male & $23(79)$ \\
\hline & Female & $6(21)$ \\
\hline Distance to anal verge (range), $\mathrm{cm}$ & & $3(0-60)$ \\
\hline Pre-operative radiotherapy $\mathrm{n}(\%)$ & & $15(52)$ \\
\hline Pre-operative chemoradiotherapy, n (\%) & & $7(24)$ \\
\hline \multirow[t]{2}{*}{ Bowel resection, $\mathrm{n}(\%)$} & Anterior & $15(52)$ \\
\hline & Abdomino-perineal excision & $14(48)$ \\
\hline Intra-operative bowel perforation, $\mathrm{n}$ & & 0 \\
\hline Tumour below peritoneal reflection, $\mathrm{n}(\%)$ & & $29(100)$ \\
\hline Complete tumour response, $\mathrm{n}(\%)$ & & $4(14)$ \\
\hline \multirow[t]{3}{*}{ Mesorectal fascia grading, n (\%) } & Mesorectal plane & $28(97)$ \\
\hline & Intramesorectal plane & $1(3)$ \\
\hline & Muscularis propria plane & 0 \\
\hline \multirow[t]{3}{*}{ Tumor differentiation, n (\%) } & Well/moderately differentiated & $22(76)$ \\
\hline & Poorly differentiated & $3(10)$ \\
\hline & Missing data & $4(14)$ \\
\hline \multirow[t]{4}{*}{ T-Stage, n (\%) } & pT0 & $5(17)$ \\
\hline & pT1 & $3(10)$ \\
\hline & pT2 & $10(35)$ \\
\hline & pT3 & $11(38)$ \\
\hline \multirow[t]{3}{*}{$\mathrm{N}-$ Stage, n (\%) } & No & $22(76)$ \\
\hline & N1 & $7(24)$ \\
\hline & $\mathrm{N} 2$ & 0 \\
\hline Lymphatic invasion, $\mathrm{n}(\%)$ & & $9(31)$ \\
\hline Venous invasion, $\mathrm{n}(\%)$ & & $11(38)$ \\
\hline Perineural invasion, $\mathrm{n}(\%)$ & & $1(3)$ \\
\hline Circumferential resection margin (range), $\mathrm{mm}$ & & $4(0.2-17)$ \\
\hline
\end{tabular}

however, the morphology did not highlight the presence of atypical cells. Further review of the patient chart revealed that at the start of the rectal surgery prior to sampling the patient was operated on with a closure of a temporary stoma.

Sample 2 displayed large numbers of inflammatory cells. In four patients, CK20/CDX2 staining was positive; however, none had positive staining for MUC2/p53. Morphological assessment was negative in all.

In the sample 3, the number of mesothelial and inflammatory cells was lower than in the other samples. There was also clear evidence of cell lysis. No cells stained positively for cancer cell markers.

Follow-up. All patients except one were followed-up for at least 24 months or until death. The median follow-up time was 36 (range=12-36) months. Adjuvant chemotherapy was administered to 18 patients $(62 \%)$, of whom five received less than $50 \%$ of the planned 6-month treatment. Three patients $(10 \%)$ developed metastases (lung metastases in two patients with simultaneous liver metastases in one). None of the patients developed peritoneal disease and there were no local recurrences.

\section{Discussion}

The double immunocytochemical staining with CK20/CDX2 and MUC2/p53 readily identified colonic cancer cells in the controls with peritoneal carcinomatosis. The method also identified tumour cells in one of the controls with sigmoidal cancer with serosal involvement. In the other control with sigmoidal cancer, the presence of free tumour cells was not shown. However, as previous studies have indicated, not all colonic cancers with serosal engagement lead to the presence of free cancer cells in peritoneal lavage (16). Furthermore, isolated cells of colonic epithelial origin can be detected with CK20/CDX2 and MUC2 staining as a result of exposure of the mucosa at the stapler line during rectal transection.

None of the patients with infraperitoneal rectal cancer had isolated cancer cells in their pelvic lavage after a TME without injuries to the MRF, underlining the importance of an undamaged MRF.

The role of local tumour spread in patients with incomplete mesorectal excision should be further evaluated in order to detect those in need of additional oncological treatment or intensified follow-up. 
A
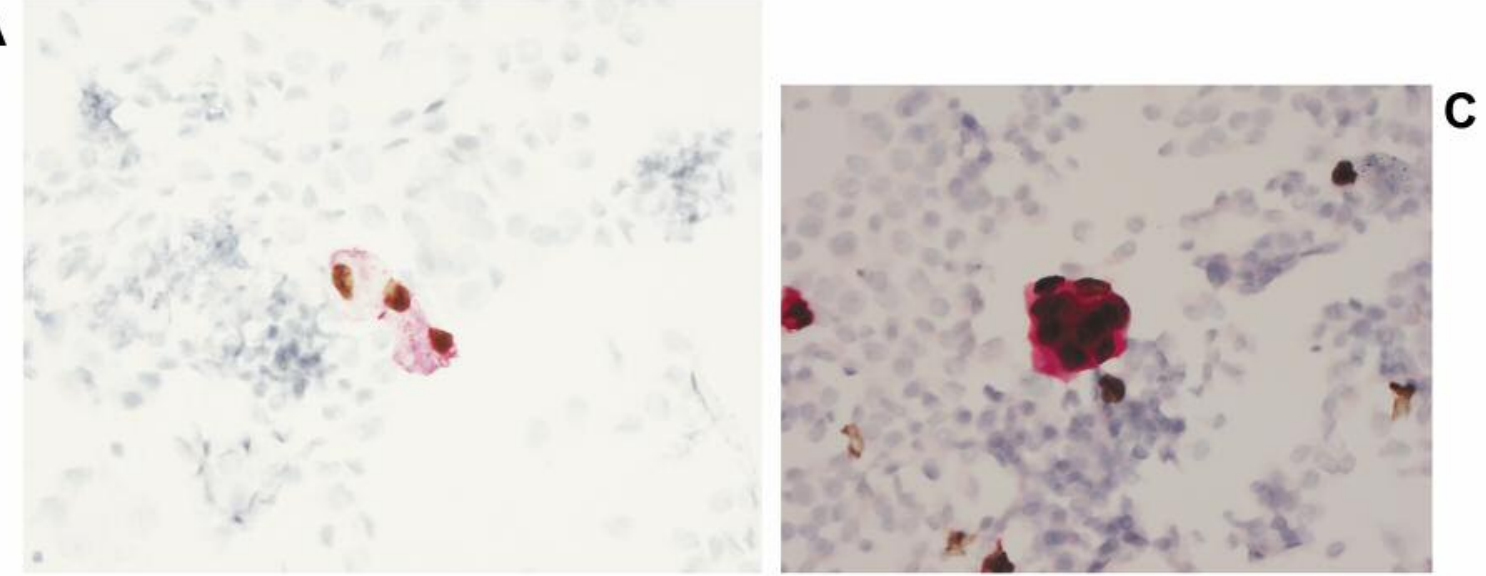

B
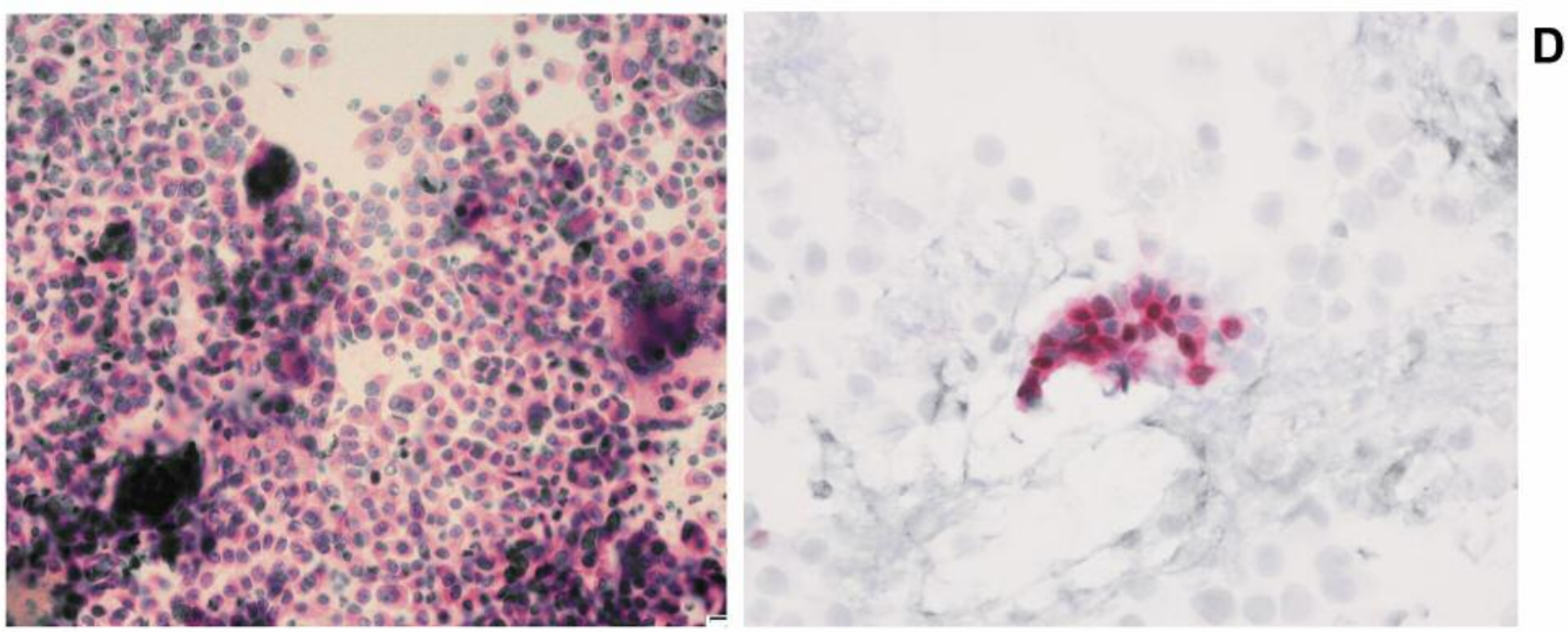

Figure 1. Immunocytochemistry of abdominal lavage. A: Sigmoidal cancer with cytokeratin 20/(CK20) caudal-type homeobox transcription factor 2 (CDX2)-positive cells. B: Atypical cells of peritoneal carcinomatosis with haematoxylin-eosin staining. C: Peritoneal carcinomatosis with CK20/CDX2-positive cells. D: Peritoneal carcinomatosis with mucin glycoprotein-2/p53-positive cells.
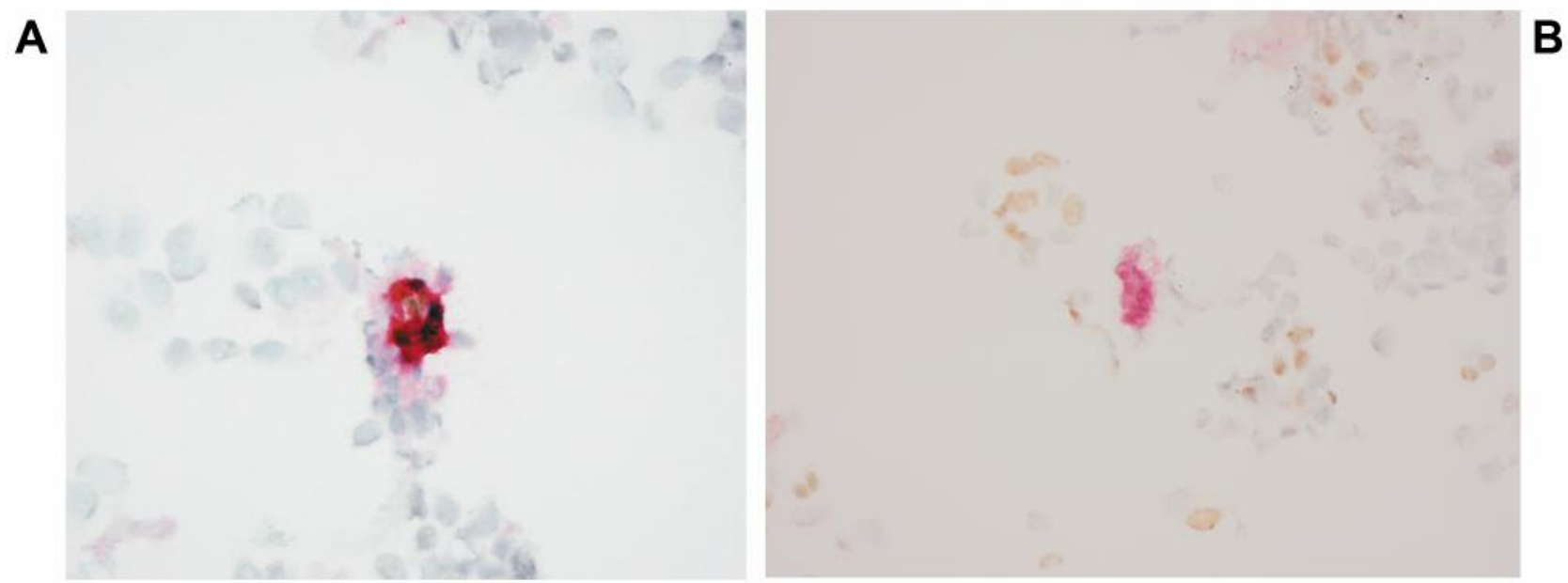

Figure 2. Immunocytochemistry of pelvic lavage. A: Colonic epithelium with cytokeratin 20/caudal-type homeobox transcription factor 2-positive cells. B: Colonic epithelium with mucin glycoprotein-2-positive cells. 
The reported differences in local recurrence after rectal cancer surgery could be explained by differences in the populations studied, or in oncological and surgical strategies (17-19). A TME of poor quality with tears and defects in the MRF increases the risk of local recurrence (1). The cause of local recurrence due to tearing/defects of the MRF can be explained by tumour spillage. However, medical oncologists have not yet included the grading of MRF involvement or infraction as a risk factor for recurrence.

As we previously reported a low frequency of local recurrences after TME for rectal cancer as a result of tailored oncological treatment and TME with excellent mesorectal grading (5), we did not expect any free cancer cells in the pelvic lavage after TME. In the present study, all specimens except one (muscularis propria plane) had a complete MRF according to the Quirke classification. In accordance with this, the double immunocytochemical method did not reveal the presence of any tumour cells.

In one patient with rectal cancer, a few MUC2-positive and CK20/CDX2-positive cells in sample 1 were observed. The origin of these cells was initially unclear. However, after a review of the surgical notes, the origin of MUC2- and CK20/CDX2-positive cells was identified as contamination by colonic cells during closure of a loop colostomy prior to sampling.

Study limitations. There was a variable amount of blood in the samples, therefore requiring different amounts of pretreatment with CytoLyt solution, possibly affecting the morphology and the antigenicity of the tumour cells $(20,21)$. We did not make any specific attempt to adjust the number of slides based on the cell density, resulting in a variable cell density in different patients. A standardization of the cell density might result in better quality slides but is timeconsuming and not applicable in routine practice. Furthermore, only one histological control for each immunocytochemical series of automated staining was used for practical reasons. Usage of a positive control for each sample is not feasible in routine practice. However, based on the positive controls, the staining method used in this study could be considered appropriate.

This double-staining method could in selected cases prove useful. For example, in cases with an incomplete TME, a positive sample could indicate the need for further oncological treatment or, in cases with locally advanced tumours with an increased risk of recurrence, be used to select those in need of intensified follow-up.

\section{Conclusion}

In conclusion, double immunocyotochemical staining of pelvic lavage is feasible. In patients with infraperitoneal rectal cancer, the pelvic lavage was negative and no free cancer cells were detected after complete TME. However, further studies are encouraged to investigate the clinical relevance in cases with free cancer cells after an incomplete TME.

\section{Contributors}

All Authors participated in data acquisition and critical revision of the article and approved the article before submission.

\section{Ethical Standards}

The study was approved by the local Ethics Committee of Uppsala University, Sweden (Dnr 2011/455) and followed the Declaration of Helsinki guidelines.

\section{Conflicts of Interest}

The Authors declare no conflict of interest in regard to this study.

\section{Acknowledgements}

This study was supported by research grants from the County of Västmanland and the Uppsala-Örebro Regional Research Council, Sweden.

\section{References}

1 Quirke P, Steele R, Monson J, Grieve R, Khanna S, Couture J, O'Callaghan C, Myint AS, Bessell E, Thompson LC, Parmar M, Stephens RJ, Sebag-Montefiore D; MRC CR07/NCIC-CTG CO16 Trial Investigators; NCRI Colorectal Cancer Study Group: Effect of the plane of surgery achieved on local recurrence in patients with operable rectal cancer: a prospective study using data from the MRC CR07 and NCIC-CTG CO16 randomised clinical trial. Lancet 373: 821-828, 2009.

2 Nagtegaal ID, Marijnen CA, Kranenbarg EK, van de Velde CJ and van Krieken JH: Circumferential margin involvement is still an important predictor of local recurrence in rectal carcinoma: not one millimeter but two millimeters is the limit. Am J Surg Pathol 26: 350-357, 2002.

3 Park JS, Huh JW, Park YA, Cho YB, Yun SH, Kim HC, Lee WY and Chun HK: A circumferential resection margin of $1 \mathrm{~mm}$ is a negative prognostic factor in rectal cancer patients with and without neoadjuvant chemoradiotherapy. Dis Colon Rectum 57: 933-940, 2014.

4 Khani MH, Smedh K and Kraaz W: Is the circumferential resection margin a predictor of local recurrence after preoperative radiotherapy and optimal surgery for rectal carcinoma? Colorectal Dis 9: 706-712, 2007.

5 Nikberg M, Kindler C, Chabok A, Letocha H, Shetye J and Smedh K: Circumferential resection margin as a prognostic marker in the modern multidisciplinary management of rectal cancer. Dis Colon Rectum 58: 275-282, 2015.

6 Nishikawa T, Watanabe T, Sunami E, Tsuno NH, Kitayama J and Nagawa H: Prognostic value of peritoneal cytology and the combination of peritoneal cytology and peritoneal dissemination in colorectal cancer. Dis Colon Rectum 52: 2016-2021, 2009. 
7 Fujii S, Shimada H, Yamagishi S, Ota M, Kunisaki C, Ike H and Ichikawa Y: Evaluation of intraperitoneal lavage cytology before colorectal cancer resection. Int J Colorectal Dis 24: 907-914, 2009.

8 Bosch B, Guller U, Schnider A, Maurer R, Harder F, Metzger U and Marti WR: Perioperative detection of disseminated tumour cells is an independent prognostic factor in patients with colorectal cancer. Br J Surg 90: 882-888, 2003.

9 Cotte E, Peyrat P, Piaton E, Chapuis F, Rivoire M, Glehen O, Arvieux C, Mabrut JY, Chipponi J, Gilly FN; EVOCAPE group: Lack of prognostic significance of conventional peritoneal cytology in colorectal and gastric cancers: results of EVOCAPE 2 multicentre prospective study. Eur J Surg Oncol 39: 707-714, 2013.

10 Kristensen AT, Wiig JN, Larsen SG, Giercksky KE and Ekstrom PO: Molecular detection (K-RAS) of exfoliated tumour cells in the pelvis is a prognostic factor after resection of rectal cancer? BMC Cancer 8: 213, 2008.

11 Mohan HM, O'Connor DB, O'Riordan JM and Winter DC: Prognostic significance of detection of microscopic peritoneal disease in colorectal cancer: a systematic review. Surg Oncol 22: e1-6, 2013.

12 Chen ZM and Wang HL: Alteration of cytokeratin 7 and cytokeratin 20 expression profile is uniquely associated with tumorigenesis of primary adenocarcinoma of the small intestine. Am J Surg Pathol 28: 1352-1359, 2004.

13 Park SY, Kim BH, Kim JH, Lee S and Kang GH: Panels of immunohistochemical markers help determine primary sites of metastatic adenocarcinoma. Arch Pathol Lab Med 131: 15611567, 2007.

14 Yantiss RK, Bosenberg MW, Antonioli DA and Odze RD: Utility of MMP-1, p53, E-cadherin, and collagen IV immunohistochemical stains in the differential diagnosis of adenomas with misplaced epithelium versus adenomas with invasive adenocarcinoma. Am J Surg Pathol 26: 206-215, 2002.
15 Purdie CA, O'Grady J, Piris J, Wyllie AH and Bird CC: p53 expression in colorectal tumors. Am J Pathol 138: 807-813, 1991.

16 Vogel P, Ruschoff J, Kummel S, Zirngibl H, Hofstadter F, Hohenberger W and Jauch KW: Prognostic value of microscopic peritoneal dissemination: comparison between colon and gastric cancer. Dis Colon Rectum 43: 92-100, 2000.

17 Khani MH and Smedh K: Centralization of rectal cancer surgery improves long-term survival. Colorectal Dis 12: 874-879, 2010.

18 Kusters M, Marijnen CA, van de Velde CJ, Rutten HJ, Lahaye MJ, Kim JH, Beets-Tan RG and Beets GL: Patterns of local recurrence in rectal cancer; a study of the Dutch TME trial. Eur J Surg Oncol 36: 470-476, 2010.

19 Wibe A, Eriksen MT, Syse A, Myrvold HE and Soreide O: Total mesorectal excision for rectal cancer - what can be achieved by a national audit? Colorectal Dis 5: 471-477, 2003.

20 Michael CW and Hunter B: Interpretation of fine-needle aspirates processed by the ThinPrep technique: cytologic artifacts and diagnostic pitfalls. Diagn Cytopathol 23: 6-13, 2000.

21 Gruchy JR, Barnes PJ and Dakin Hache KA: CytoLyt (R) fixation and decalcification pretreatments alter antigenicity in normal tissues compared with standard formalin fixation. Appl Immunohistochem Mol Morphol 23: 297-302, 2015.
Received February 19, 2017

Revised March 13, 2017

Accepted March 20, 2017 